\title{
Homoleptic \\ Cyclic \\ Trinuclear \\ d 10 \\ Complexes: from Self-Association via Metallophilic and Excimeric Bonding to the Breakage Thereof via Oxidative Addition, Dative Bonding, Quadrupolar, and Heterometal Bonding Interactions
}

Rossana Galassi ,1 Manal A. Rawashdeh-Omary ,2H. V. Rasika Dias , 3 and Mohammad A.

Omary 4

${ }_{1}$ School of Science and Technology, University of Camerino, Camerino, Italy

${ }_{2}$ Department of Chemistry and Physics, Texas Woman's University, Denton, Texas,

USA

3Department of Chemistry and Biochemistry, The University of Texas at Arlington,

Arlington, Texas, USA

${ }_{4}$ Department of Chemistry, University of North Texas, Denton, Texas, USA

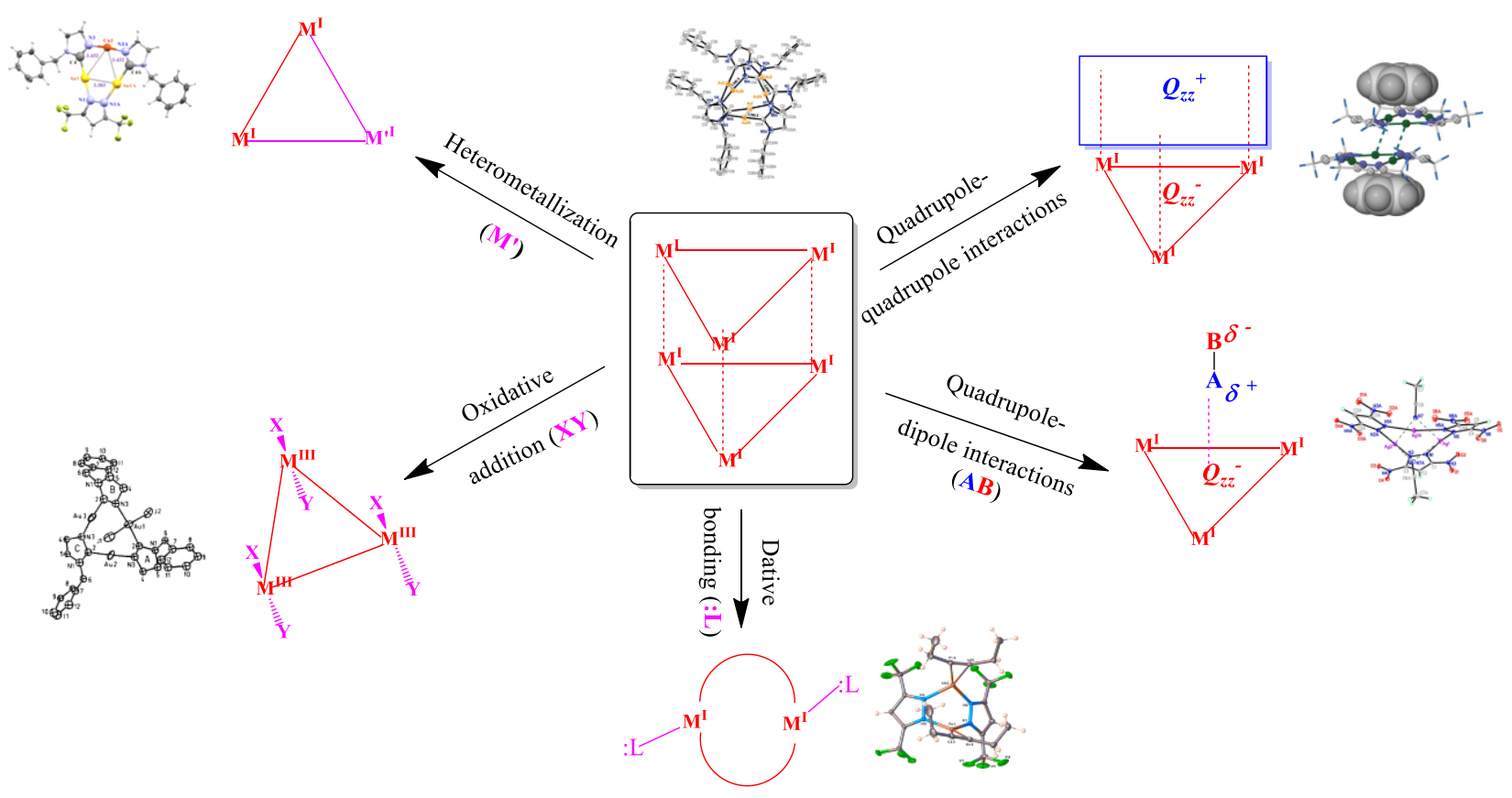

